International Journal of Pure and Applied Mathematics

Volume 104 No. 1 2015, 107-117

ISSN: 1311-8080 (printed version); ISSN: 1314-3395 (on-line version)

url: http://www.ijpam.eu

doi: http://dx.doi.org/10.12732/ijpam.v104i1.9

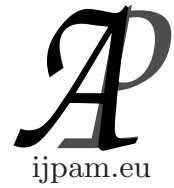

\title{
HYERS-ULAM STABILITY OF SOME FREDHOLM INTEGRAL EQUATION
}

\author{
Liubin Hua ${ }^{1}$, Jinghao Huang ${ }^{2}$, Yongjin $\mathrm{Li}^{3} \S$ \\ ${ }^{1}$ Guangzhou Sontan Polytechnic College \\ Guangzhou, 511370, P.R. CHINA \\ ${ }^{2,3}$ Department of Mathematics \\ Sun Yat-Sen University \\ Guangzhou, 510275 P.R. CHINA
}

\begin{abstract}
We prove the Hyers-Ulam stability of some kinds of Fredholm integral equation. That is, if $\phi(t)$ is an approximate solution of a Fredholm integral equation, then there exists an exact solution of the differential equation near to $\phi(t)$.
\end{abstract}

AMS Subject Classification: 34K20, 26D10

Key Words: Hyers-Ulam stability, Fredholm integral equation, Fourier transform

\section{Introduction and Preliminaries}

S.M. Ulam [15] gave a wide-ranging talk about a series of important unsolved problems in 1940. The question concerning the stability of group homomorphisms is one of them. D.H. Hyers (see [1]) proved the stability for the case of approximately additive mappings under the assumption that $G_{1}$ and $G_{2}$ are Banach spaces. After then, the Hyers-Ulam stability of function equation (see $[2,11,12,13])$ and differential function (see $[3,4,6,7,8,9,10,14])$ was investigated by several mathematicians.

Received: May 20, 2015

(C) 2015 Academic Publications, Ltd.

$\S$ Correspondence author url: www.acadpubl.eu 
Fredholm integral equation is a kind of important integral equation. In this paper, we will try to investigate the Hyers-Ulam stability to some kinds of Fredholm integral equation:

The first class of convolutional Fredholm integral

$$
\phi(t)=f(t)+\int_{-\infty}^{+\infty} k(t-s) \phi(s) d s
$$

and the second class of Fredholm integral

$$
\int_{-\infty}^{+\infty} k(t-s) \phi(s) d s=f(t)
$$

and

$$
\int_{-\infty}^{+\infty} k(t+s) \phi(s) d s=f(t)
$$

where the kernel of integral $k(t)$ is continuous and satisfies some properties, and $f(t)$ is continuous.

Firstly, we should introduce these prerequisite knowledge.

Fourier transform:

$$
F(f)(s)=F(s)=\frac{1}{\sqrt{2 \pi}} \int_{-\infty}^{+\infty} f(t) e^{-i s t} d t
$$

Inverse Fourier transform:

$$
F^{-1}[F](t)=f(t)=\frac{1}{\sqrt{2 \pi}} \int_{-\infty}^{+\infty} F(s) e^{i s t} d s
$$

and we know that Fourier transform has some properties:

(a) $F\left[\alpha f_{1}+\beta f_{2}\right]=\alpha F\left[f_{1}\right]+\beta F\left[f_{2}\right] \quad \forall \alpha, \beta \in \mathbb{C}$;

(b) $F\left[f^{\prime}(t)\right]=i s F[f(t)]$;

(c) $F^{-1}[F(s)](t)=f(t)$;

(d) Suppose that $h(t)=\int_{0}^{t} f(x) d x$, then $F[h(t)]=\frac{1}{i s} F[f(t)]$;

(e) Suppose that

$$
h(t)=\frac{1}{\sqrt{2 \pi}} \int_{-\infty}^{+\infty} f(t-x) g(x) d x=\frac{1}{\sqrt{2 \pi}} \int_{-\infty}^{+\infty} g(t-x) f(x) d x,
$$

we have

$$
H(s)=F(h(t))=F[f] \cdot F[g]=F(s) \cdot G(s)
$$


(f) $F[f(t-a)]=e^{-i a s} F[f(t)]$;

(g) $\int_{-\infty}^{+\infty}|F(f)|^{2} d s=\int_{-\infty}^{+\infty}|f(x)|^{2} d x$.

And the following inequality is useful to our proof to the main results.

Theorem 1.1 (Hölder's Inequality). Assume that $p>1, \frac{1}{p}+\frac{1}{q}=1, x \in$ $L^{p}(E), y \in L^{q}(E)$, then $x y \in L(E)$ and

$$
\int_{E}|x(t) y(t)| d t \leq\left(\int_{E}\left|x(t)^{p}\right| d t\right)^{\frac{1}{p}}\left(\int_{E}|y(t)|^{q} d t\right)^{\frac{1}{q}} .
$$

\section{Main Results}

Theorem 2.1. Suppose that $f(t), k(t)$ are real continuous functions and $f(t), k(t) \in L^{2}(-\infty,+\infty), K(s) \neq \frac{\downarrow 1}{2 \pi}$ and $\frac{v}{1-\frac{1}{2 \pi} K(s)} \in L^{2}(-\infty,+\infty)$, and if a real function $\phi(t): \mathbb{R} \rightarrow \mathbb{R}$ and $F(\phi)(s)$ exists, satisfies that

$$
\left\|\phi(t)-f(t)-\int_{-\infty}^{+\infty} k(t-s) \phi(s) d s\right\| \leq \varphi(t)
$$

where $\varphi(t) \in L^{2}(-\infty,+\infty), \varphi(t) \geq 0$. Then there exists a unique real solution $\phi_{1}(t)\left(F\left(\phi_{1}\right)(s)\right.$ exists) satisfies Eq.(1.1) and $\left\|\phi-\phi_{1}\right\| \leq M\left(\int_{-\infty}^{+\infty} \varphi(x)^{2} d x\right)^{\frac{1}{2}}$ where $M$ is a constant.

Proof. (1)Firstly, we will prove the stability.

Let $\phi$ be a solution of Ineq.(2.1) and put

$$
\phi(t)-f(t)-\int_{-\infty}^{+\infty} k(t-s) \phi(s) d s:=g(t)
$$

where $|g(t)| \leq \varphi(t)$, since $\varphi(t) \in L^{2}(-\infty,+\infty)$, we know that $g(t) \in L^{2}(-\infty,+\infty)$.

It follows Fourier transform and the property(e) that

$$
\Phi(s)=F(s)+G(s)+\sqrt{2 \pi} K(s) \Phi(s) .
$$

Then we have

$$
\Phi(s)=\frac{F(s)+G(s)}{1-\sqrt{2 \pi} K(s)} .
$$


It follows the inverse Fourier transform that

$$
p(t)=\frac{1}{\sqrt{2 \pi}} \int_{-\infty}^{+\infty} \frac{1}{1-\sqrt{2 \pi} K(s)} e^{i s t} d s .
$$

Then, we can know from the property (a) and (e) that

$$
\Phi(s)=(F(s)+G(s)) \cdot P(s)=F\left[\frac{1}{\sqrt{2 \pi}} \int_{-\infty}^{+\infty}(f(t-x)+g(t-x)) p(x) d x\right](s) .
$$

It follows the inverse Fourier transform and properties (c) that

$$
\begin{aligned}
\phi(t) & =\frac{1}{\sqrt{2 \pi}} \int_{-\infty}^{+\infty}(f(t-x)+g(t-x)) p(x) d x \\
& =\frac{1}{2 \pi} \int_{-\infty}^{+\infty} \int_{-\infty}^{+\infty}(f(t-x)+g(t-x)) \frac{1}{1-\sqrt{2 \pi} K(s)} e^{i s x} d s d x,
\end{aligned}
$$

and if we let $g(t)=0$, we get a solution of Eq. (1.1)

$$
\phi_{1}(t)=\frac{1}{2 \pi} \int_{-\infty}^{+\infty} \int_{-\infty}^{+\infty} f(t-x) \frac{1}{1-\sqrt{2 \pi} K(s)} e^{i s x} d s d x
$$

Then, follows the Theorem 1.1

$$
\begin{aligned}
\left|\phi(t)-\phi_{1}(t)\right|= & \frac{1}{2 \pi}\left|\int_{-\infty}^{+\infty} \int_{-\infty}^{+\infty} g(t-x) \frac{1}{1-\sqrt{2 \pi} K(s)} e^{i s x} d s d x\right| \\
\leq & \frac{1}{2 \pi}\left(\int_{-\infty}^{+\infty} \varphi(t-x)^{2} d x\right)^{\frac{1}{2}} \\
& \times\left(\int_{-\infty}^{+\infty}\left|\int_{-\infty}^{+\infty} \frac{1}{1-\sqrt{2 \pi} K(s)} e^{i s x} d s\right|^{2} d x\right)^{\frac{1}{2}} .
\end{aligned}
$$

Moreover, following property (g), we know that

$$
\begin{aligned}
& \frac{1}{\sqrt{2 \pi}}\left(\int_{-\infty}^{+\infty}\left|\frac{1}{\sqrt{2 \pi}} \int_{-\infty}^{+\infty} \frac{1}{1-\sqrt{2 \pi} K(s)} e^{i s x} d s\right|^{2} d x\right)^{\frac{1}{2}} \\
& =\frac{1}{\sqrt{2 \pi}}\left(\int_{-\infty}^{+\infty}\left|\frac{1}{1-\sqrt{2 \pi} K(x)}\right|^{2} d x\right)^{\frac{1}{2}}=M<\infty \text {. }
\end{aligned}
$$

Therefore $\left|\phi(t)-\phi_{1}(t)\right| \leq M\left(\int_{-\infty}^{+\infty} \varphi(x)^{2} d x\right)^{\frac{1}{2}}$.

(2) Now, we will prove the existence and uniqueness of Eq.(1.1), and that the solution is real function. 
Existence. Let $f \in L^{2}(-\infty,+\infty)$, follows the property (g), we know that $F \in L^{2}(-\infty,+\infty)$, and $\frac{\sqrt{ }}{1-\frac{1}{2 \pi} K(s)} \in L^{2}(-\infty,+\infty)$, such that

$$
\int_{-\infty}^{+\infty}\left|\frac{F(s)}{1-\sqrt{2 \pi} K(s)}\right| d s \leq\left.\left.\left.\left.\left|\int_{-\infty}^{+\infty}\right| F(s)\right|^{2} d s\right|^{\frac{1}{2}}\left|\int_{-\infty}^{+\infty}\right| \frac{1}{1-\sqrt{2 \pi} K(s)}\right|^{2} d s\right|^{\frac{1}{2}} .
$$

Thus $\left|\frac{F F(s)}{1-\frac{2 \pi}{2 \pi} K(s)}\right| \in L(-\infty,+\infty)$, and the inverse fourier transform of $\frac{\sqrt{F(s)}}{1-\frac{2 \pi}{2 \pi} K(s)}$ exists, that means $\phi_{1}(t)$ is actually a solution of Eq. (1.1).

Uniqueness. If there exists another solution $\tilde{\phi}_{1}(t)$ satisfies Eq.(1.1) and the fourier transform of it exists. By using fourier transform, we have

$$
\tilde{\Phi}_{1}(s)=\frac{F(s)+G(s)}{1-\sqrt{2 \pi} K(s)}=\Phi_{1}(s)
$$

follows the property (c), we know $\tilde{\phi}_{1}(t)=\phi_{1}(t)$.

Real function. Since $f, k$ are real functions, we know it is impossible that the solution of Eq.(1.1) $\phi_{1}$ is a pure imaginary function. If $\phi_{1}$ is a complex function, from the above proof we know that $\phi_{1}(t)=\Re \phi_{1}(t)+i \Im\left(\phi_{1}(t)\right)$ and fourier transform of $\Re\left(\phi_{1}(t)\right)$ and $\Im\left(\phi_{1}(t)\right)$ exist, and $\Im\left(\phi_{1}(t)\right)$ is a solution of

$$
\Im\left(\phi_{1}(t)\right)=\int_{-\infty}^{+\infty} k(t-s) \Im\left(\phi_{1}(t)\right) d s
$$

by fourier transform and property(e)

$$
F\left(\Im\left(\phi_{1}(s)\right)\right)=\sqrt{2 \pi} K(s) F\left(\Im\left(\phi_{1}(s)\right)\right)
$$

and $K(s) \neq \frac{1}{2 \pi}$, such that $F\left(\Im\left(\phi_{1}(s)\right)\right)=0=F\left(\Im\left(\phi_{1}(s)\right)\right)$. Thus, $\phi(t)_{1}$ is a real function.

Theorem 2.2. Suppose that $f(t), k(t)$ are real continuous functions and $f(t), k(t) \in L^{2}(-\infty,+\infty), K(s) \neq 0$ and $\frac{1}{K(s)} \in L^{2}(-\infty,+\infty)$, and and if a real function $\phi(t): \mathbb{R} \rightarrow \mathbb{R}$ and $F(\phi)(s)$ exists, satisfies that

$$
\left\|f(t)-\int_{-\infty}^{+\infty} k(t-s) \phi(s) d s\right\| \leq \varphi(t)
$$

where $\varphi(t) \in L^{2}(-\infty,+\infty), \varphi(t) \geq 0$. Then there exists a unique real solution $\phi_{1}(t)\left(F\left(\phi_{1}\right)(s)\right.$ exists) satisfies Eq.(1.2) and $\left\|\phi-\phi_{1}\right\| \leq M\left(\int_{-\infty}^{+\infty} \varphi(x)^{2} d x\right)^{\frac{1}{2}}$ where $M$ is a constant. 
Proof. (1)Firstly, we will prove the stability.

Let $\phi$ be a solution of Ineq.(2.7) and put

$$
f(t)-\int_{-\infty}^{+\infty} k(t-s) \phi(s) d s:=g(t)
$$

where $|g(t)| \leq \varphi(t)$, since $\varphi(t) \in L^{2}(-\infty,+\infty)$, we know that $g(t) \in L^{2}(-\infty,+\infty)$.

It follows Fourier transform and the property $(\mathrm{e})$ that

$$
F(s)-\sqrt{2 \pi} K(s) \Phi(s)=G(s) .
$$

Then we have

$$
\Phi(s)=\frac{F(s)-G(s)}{\sqrt{2 \pi} K(s)} .
$$

It follows the inverse Fourier transform that

$$
p(t)=\frac{1}{\sqrt{2 \pi}} \int_{-\infty}^{+\infty} \frac{1}{\sqrt{2 \pi} K(s)} e^{i s t} d s .
$$

Then, we can know from the property(a) and (e) that

$$
\Phi(s)=(F(s)-G(s)) \cdot P(s)=F\left[\frac{1}{\sqrt{2 \pi}} \int_{-\infty}^{+\infty}(f(t-x)-g(t-x)) p(x) d x\right](s) .
$$

It follows the inverse Fourier transform and properties(c) that

$$
\begin{aligned}
\phi(t) & =\frac{1}{\sqrt{2 \pi}} \int_{-\infty}^{+\infty}(f(t-x)-g(t-x)) p(x) d x \\
& =\frac{1}{(2 \pi)^{\frac{3}{2}}} \int_{-\infty}^{+\infty} \int_{-\infty}^{+\infty}(f(t-x)-g(t-x)) \frac{1}{K(s)} e^{i s x} d s d x
\end{aligned}
$$

and if we let $g(t)=0$, we get a solution of Eq.(1.2)

$$
\phi_{1}(t)=\frac{1}{(2 \pi)^{\frac{3}{2}}} \int_{-\infty}^{+\infty} \int_{-\infty}^{+\infty} f(t-x) \frac{1}{K(s)} e^{i s x} d s d x
$$

Therefore (using Theorem 1.1):

$$
\begin{aligned}
\left|\phi(t)-\phi_{1}(t)\right| & =\frac{1}{(2 \pi)^{\frac{3}{2}}}\left|\int_{-\infty}^{+\infty} \int_{-\infty}^{+\infty} g(t-x) \frac{1}{K(s)} e^{i s x} d s d x\right| \\
& \leq \frac{1}{(2 \pi)^{\frac{3}{2}}}\left(\int_{-\infty}^{+\infty} \varphi(t-x)^{2} d x\right)^{\frac{1}{2}}\left(\int_{-\infty}^{+\infty}\left|\int_{-\infty}^{+\infty} \frac{1}{K(s)} e^{i s x} d s\right|^{2} d x\right)^{\frac{1}{2}}
\end{aligned}
$$


Moreover, following property (g), we have

$$
\frac{1}{2 \pi}\left(\int_{-\infty}^{+\infty}\left|\frac{1}{\sqrt{2 \pi}} \int_{-\infty}^{+\infty} \frac{1}{K(s)} e^{i s x} d s\right|^{2} d x\right)^{\frac{1}{2}}=\frac{1}{2 \pi}\left(\int_{-\infty}^{+\infty}\left|\frac{1}{K(x)}\right|^{2} d x\right)^{\frac{1}{2}}=M<\infty .
$$

Therefore $\left|\phi(t)-\phi_{1}(t)\right| \leq M\left(\int_{-\infty}^{+\infty} \varphi(x)^{2} d x\right)^{\frac{1}{2}}$.

(2) Now, we will prove the existence and uniqueness of Eq.(1.2), and the solution is actually a real function.

Existence. $f \in L^{2}(-\infty,+\infty)$, follows the property(g), we know that $F \in$ $L^{2}(-\infty,+\infty)$. And $\frac{1}{K(s)} \in L^{2}(-\infty,+\infty)$, following the proof of theorem 2.1, we can know that $\phi_{1}(s)$ exist and satisfy Eq.(1.2).

Uniqueness. If there exists another solution $\tilde{\phi}_{1}(t)$ satisfies Eq.(1.2) and the fourier transform of it exists, by using fourier transform, by using fourier transform, we have

$$
\tilde{\Phi}_{1}(s)=\frac{F(s)-G(s)}{\sqrt{2 \pi} K(s)}=\Phi_{1}(s)
$$

follows the property $(\mathrm{c})$, we know $\tilde{\phi}_{1}(t)=\phi_{1}(t)$.

Real function. Since $f, k$ are real functions, we know it is impossible that the solution of Eq.(1.2) $\phi_{1}$ is a pure imaginary function. If $\phi_{1}$ is a complex function, from the above proof we know that $\phi_{1}(t)=\Re \phi_{1}(t)+i \Im\left(\phi_{1}(t)\right)$ and fourier transform of $\Re\left(\phi_{1}(t)\right)$ and $\Im\left(\phi_{1}(t)\right)$ exist, and $\Im\left(\phi_{1}(t)\right)$ is a solution of

$$
0=\int_{-\infty}^{+\infty} k(t-s) \Im\left(\phi_{1}(t)\right) d s
$$

by fourier transform and property(e)

$$
0=K(s) \Im\left(\Phi_{1}(s)\right)
$$

and $K(s) \neq 0$, such that $\Im\left(\Phi_{1}(s)\right)=0=\Im\left(\phi_{1}(t)\right)$. Thus, $\phi_{1}(t)$ is a real function.

Theorem 2.3. Suppose that $f(t), k(t)$ are real continuous functions and $f(t), k(t) \in L^{2}(-\infty,+\infty), K(s) \neq 0$ and $\frac{1}{K(s)} \in L^{2}(-\infty,+\infty)$, and and if a real function $\phi(t): \mathbb{R} \rightarrow \mathbb{R}$ and $F(\phi)(s)$ exists, satisfies that

$$
\left\|f(t)-\int_{-\infty}^{+\infty} k(t+s) \phi(s) d s\right\| \leq \varphi(t)
$$


where $\varphi(t) \in L^{2}(-\infty,+\infty), \varphi(t) \geq 0$. Then there exists a unique real solution $\phi_{1}(t)\left(F\left(\phi_{1}\right)(s)\right.$ exists) satisfies Eq.(1.3) and $\left\|\phi-\phi_{1}\right\| \leq M\left(\int_{-\infty}^{+\infty} \varphi(x)^{2} d x\right)^{\frac{1}{2}}$ where $M$ is a constant.

Proof. (1) We will prove the stability property.

Let $\phi$ be a solution of Ineq.(2.13) and put

$$
f(t)-\int_{-\infty}^{+\infty} k(t+s) \phi(s) d s:=g(t),
$$

where $|g(t)| \leq \varphi(t)$, since $\varphi(t) \in L^{2}(-\infty,+\infty)$, we know that $g(t) \in L^{2}(-\infty,+\infty)$.

It follows, using Fourier transform, that

$$
\begin{aligned}
\int_{-\infty}^{+\infty} f(t) e^{-i s t} d t= & \int_{-\infty}^{+\infty} g(t) e^{-i s t} d t+\int_{-\infty}^{+\infty}\left[\int_{-\infty}^{+\infty} k(t+x) \phi(x) d x\right] e^{-i s t} d t \\
= & \int_{-\infty}^{+\infty} g(t) e^{-i s t} d t+\int_{-\infty}^{+\infty} \int_{-\infty}^{+\infty} k(t+x) e^{-i s(t+x)} \phi(x) \\
& \times e^{i s x} d x d t, \\
& F(s)-G(s)=\sqrt{2 \pi} K \cdot F^{-1}(\phi(t))(s) .
\end{aligned}
$$

Then we have

$$
\phi(t)=F\left(\frac{F-G}{\sqrt{2 \pi} K}\right)
$$

and if we let $g(t)=0$, we obtain solution of Eq.(1.3)

$$
\phi_{1}(t)=F\left(\frac{F}{\sqrt{2 \pi} K}\right) .
$$

Thus

$$
\begin{aligned}
\left|\phi(t)-\phi_{1}(t)\right| & =\left|F\left(\frac{G}{\sqrt{2 \pi} K}\right)\right| \\
& \leq \frac{1}{2 \pi}\left|\int_{-\infty}^{+\infty} G(s)^{2} d s\right|^{\frac{1}{2}}\left|\int_{-\infty}^{+\infty}\left(\frac{e^{-i s t}}{K(s)}\right)^{2} d s\right|^{\frac{1}{2}} \\
& \leq \frac{1}{2 \pi}\left|\int_{-\infty}^{+\infty} \varphi(s)^{2} d s\right|^{\frac{1}{2}}\left|\int_{-\infty}^{+\infty}\left(\frac{e^{-i s t}}{K(s)}\right)^{2} d s\right|^{\frac{1}{2}} \\
& \leq \frac{1}{2 \pi}\left|\int_{-\infty}^{+\infty} \varphi(s)^{2} d s\right|^{\frac{1}{2}}\left|\int_{-\infty}^{+\infty}\left(\frac{1}{K(s)}\right)^{2} d s\right|^{\frac{1}{2}}
\end{aligned}
$$


and let $M=\frac{1}{2 \pi}\left|\int_{-\infty}^{+\infty}\left(\frac{1}{K(s)}\right)^{2} d s\right|^{\frac{1}{2}}$, we have ||$\phi-\phi_{1}|| \leq M\left|\int_{-\infty}^{+\infty} \varphi(s)^{2} d s\right|^{\frac{1}{2}}$.

(2) Now, we will prove the existence and uniqueness of Eq.(1.3), and the solution is actually a real function.

Existence. $f \in L^{2}(-\infty,+\infty)$, follows the property(g), we know that $F \in$ $L^{2}(-\infty,+\infty)$. And $\frac{1}{K(s)} \in L^{2}(-\infty,+\infty)$, following the proof of theorem 2.1, we can know that $\phi_{1}(s)$ exist and satisfy Eq.(1.3).

Uniqueness. If there exists another real solution $\tilde{\phi}_{1}(t)$ satisfies Eq.(1.3) and the fourier transform of it exists, by using fourier transform, we have

$$
F^{-1}\left(\tilde{\phi}_{1}(s)\right)=\frac{F}{\sqrt{2 \pi} K}=F^{-1}\left(\phi_{1}(s)\right)
$$

follows the property $(\mathrm{c})$, we know $\tilde{\phi}_{1}(t)=\phi_{1}(t)$.

Real function. Since $f, k$ are real functions, we know it is impossible that the solution of Eq.(1.3) $\phi_{1}$ is a pure imaginary function. If $\phi_{1}$ is a complex function, from the above proof we know that $\phi_{1}(t)=\Re \phi_{1}(t)+i \Im\left(\phi_{1}(t)\right)$ and Fourier transform of $\Re\left(\phi_{1}(t)\right)$ and $\Im\left(\phi_{1}(t)\right)$ exist, and $\Im\left(\phi_{1}(t)\right)$ is a solution of

$$
0=\int_{-\infty}^{+\infty} k(t+s) \Im\left(\phi_{1}(t)\right) d s,
$$

by Fourier transform:

$$
0=\sqrt{2 \pi} K(s) \cdot F^{-1}\left(\Im\left(\phi_{1}(t)\right)\right)(s)
$$

and $K(s) \neq 0$, such that $\Im\left(\Phi_{1}(s)\right)=0=\Im\left(\phi_{1}(t)\right)$. Thus, $\phi_{1}(t)$ is a real function.

\section{Acknowledgments}

The authors would like to thank the anonymous referee for his or her suggestions and corrections.

This work was supported by the National Natural Science Foundation of P.R. China (10871213). 


\section{References}

[1] D. H. Hyers, On the stability of the linear functional equation, Proc. Nat. Acad. Sci. U.S.A. 27 (1941) 222-224.

[2] K. -W. Jun and Y. -H. Lee, A generalization of the Hyers-Ulam-Rassias stability of Jensen's equation, J. Math. Anal. Appl. 238 (1999), 305-315.

[3] S. -M. Jung, Hyers-Ulam stability of linear differential equations of first order, Appl. Math. Lett. 17 (2004), 1135-1140.

[4] S. -M. Jung, Hyers-Ulam stability of linear differential equations of first order (II), Appl. Math. Lett. 19 (2006), 854-858.

[5] Y. Li, Hyers-Ulam stability of linear differential equations $y^{\prime \prime}=\lambda^{2} y$. Thai J. Math. 8 (2010), no. 2, 215-219.

[6] Y. Li, Y. Shen, Hyers-Ulam stability of linear differential equations of second order, Appl. Math. Lett. 23 (2010), 306-309.

[7] T. Miura, On the Hyers-Ulam stability of a differentiable map, Sci. Math. Japan 55 (2002), 17-24.

[8] T. Miura, S. -M. Jung, S. -E. Takahasi, Hyers-Ulam-Rassias stability of the Banach space valued linear differential equations $y^{\prime}=\lambda y$, J. Korean Math. Soc. 41 (2004), 995-1005.

[9] M. Obłoza, Hyers stability of the linear differential equation, Rocznik Nauk.-Dydakt. Prace Mat. No. 13. (1993), 259-270.

[10] M. Obłoza, Connections between Hyers and Lyapunov stability of the ordinary differential equations, Rocznik Nauk.-Dydakt. Prace Mat. No. 14(1997), 141-146.

[11] C. -G. Park, On the stability of the linear mapping in Banach modules, J.Math. Anal. Appl. 275 (2002), 711-720.

[12] Th. M. Rassias, On the stability of linear mapping in Banach spaces, Proc. Amer. Math. Soc. 72 (1978), 297-300.

[13] Th. M. Rassias, On the stability of functional equations and a problem of Ulam, Acta Appl. Math. 62 (2000), 23-130. 
[14] S. -E. Takahasi, T. Miura, S. Miyajima, On the Hyers-Ulam stability of the Banach space-valued differential equation $y^{\prime}=\lambda y$, Bull. Korean Math. Soc. 39 (2002), 309-315.

[15] S. M. Ulam, A Collection of the Mathematical Problems, Interscience, New York, 1960. 
